\title{
Editorial
}

\section{Let's take the next step in documentation}

W ith increasing number of article submissions from our part of the world it is heartening to note that we are slowly but surely getting transformed from "do only" mode to "do and document" mode. But everything is not hunky dory when as editor of IJPS I look into my kitty of papers. I am finding that more and more young colleagues are turning impatient and after treating a patient rather well, converting their experience into a small and insignificant case report. This was perhaps acceptable a decade ago, but today a lot more is expected of us. What we consider to be rare and unique gets labeled as banal and mundane by the reviewers and is rejected.

Most case reports are on one of six topics:

- An unexpected association between diseases or symptoms.

- An unexpected event in the course of observing or treating a patient.

- Findings that shed new light on the possible pathogenesis of a disease or adverse effect.

- Unique or rare features of the disease.

- Unique therapeutic approaches.

- A positional or quantitative variation of the anatomical structures.

A case report is a type of anecdotal evidence. As such, it is less scientifically rigorous than controlled clinical data involving a larger sample size. Case reports permit discovery of new diseases and unexpected effects (adverse or beneficial) as well as the study of mechanisms, and have a high sensitivity for detecting novelty and, therefore, remain one of the cornerstones of medical progress. Sigmund Freud, Christiaan Barnard, William

\begin{tabular}{|l|l|}
\hline \multicolumn{2}{|c|}{ Access this article online } \\
\hline Quick Response Code: & Website: \\
\hline & www.ijps.org \\
\cline { 2 - 2 } & Dol: \\
\hline
\end{tabular}

MacIntire all reported their path-breaking work in case reports. Case reports provide many new ideas but how can science be advanced with the report of yet another Goldenhar syndrome or Van der Woude syndrome? How can we try to report yet another platysma flap if we are not offering a newer dimension?

Case reports are not considered as research particularly in this day and age of evidence-based research and practice. They are considered level 5 or at the best level 4 evidence and hence do not get much importance. Future authors do not cite them, and journals are reluctant to carry them as the pull down their impact factor.

Reviewers and editorial board members are quite justifiably pushing the authors towards evidence-based surgery and toward ethical research practices. They are asking us to use both individual clinical expertise and the best available external evidence in the form of prior publications in order to treat our patients as neither alone is enough. Without clinical expertise, they feel we will be tyrannized by external evidence, for even excellent external evidence may be inapplicable to or inappropriate for an individual patient. The Asian skin scars differently and Wolfe grafts on face invariably hyper pigment, no matter what published literature from the West says! Then again without current best external evidence, we risk becoming rapidly out of date, to the detriment of our patients!

Every word that is published in journals cannot be considered as gospel truth and hence to appraise their trustworthiness all published work is assigned a level of evidence. ${ }^{[1]}$ These are:

Level 1: Consists of randomized controlled trials (RCTs) and systematic reviews of all RCT data including metaanalyses.

Level 2: Consists of cohort studies and their systematic reviews.

Level 3: Consists of case control studies and their systematic reviews.

Indian Journal of Plastic Surgery May-August 2014 Vol 47 Issue 2 
Level 4: Consists of case-series.

Level 5: Consists of expert opinion without explicit critical appraisal, or based on physiology, bench research or 'first principles'.

There is very little level 1 evidence in plastic surgery literature, but those which exist have changed lives. The Cochrane Injuries Group published a systematic review of RCTs of the administration of human albumin to critically illpatients, in 1998. This was of relevance to all plastic surgeons who treat burn injuries since it suggested that there was an increase in mortality of $6 \%$ in critically ill-patients who had been treated with human albumin, compared with patients who had received either crystalloids or no treatment. ${ }^{[2]}$ This is the value of level 1 evidence.

Well-designed research studies have led to the near disappearance of previously common surgical procedures such as extracranial - intracranial bypass for stroke prevention, internal mammary artery ligation for heart disease and vagotomy-gastro-jujunostomy for peptic ulcer disease. However, all this has been possible because someone chose to question what was traditional then, and designed an ethical model of research which had:

1. Respect for autonomy: The patients' right to selfdetermination and decision making.

2. Non-maleficence: Avoiding the causation of harm or primum non nocere.

3. Beneficence: Acting in a way that benefits the patient after balancing the potential risks and harms.

4. Justice: Treating patients in similar positions in a similar manner by distributing risks and costs fairly. ${ }^{[3]}$

Research in plastic, reconstructive and aesthetic surgery usually starts with a spark, a bright idea. Ponten thought of including the deep fascia in his leg flaps and increased the viable dimensions exponentially! Did he have a prior protocol approved by his hospital ethical committee? At which stage did he take his research to the ethical committee? Did he at all? Because there are no guidelines, which define threshold levels of clinical innovation or acceptable added risk, the responsibility for judging when a new or novel surgical practice becomes research rests solely with the individual surgeon. Unlike drugs, medical devices and other products that require demonstration of safety and effectiveness by laboratory tests and animal studies before approval by authorities like the Food and Drug Administration, there are no regulations describing how new surgical procedures should be tested. Here is where the human in the surgeon needs to take the call. N-butyl cyanoacrylate is a pharmacological product, used as tissue adhesive and $\mathrm{N}$-methyl cyano acrylate is an industrial product used to, as the makers claim, mend everything except broken hearts. Though almost 500 times more economical, is it ethical to use the industrial product to adhere skin grafts? Can you blame my reviewers if they raise a hysterical fit when someone tries to publish this misadventure?

If we do not get our act together now and take the next step towards better and more ethical documentation, we stand to miss out big time! You have been a surgeon for many years. For the past couple of them, if you are not doing something new every now and then your performance in the operating room may have reached a plateau. I'd like to think it's a good thing - you've arrived at your professional peak. But when it comes to your style of documentation to me it seems as if you've just stopped getting better. This is dangerous. Collect your data with patience, analyze them with great dedication, think out of the box and see how else can the same data be interpreted? Ask a junior colleague to put his mind to the task and see if he can think something that you were missing all throughout. Study the published literature and see if you get that spark of creativity. Then write an original article. Yes, it is far more difficult than writing a case report, but it is also far more satisfying. And even more satisfying would be ethically designing a multi-centric randomized control trial on managing maxillo-facial trauma, hand trauma, vascular or neurological trauma, cleft lip surgery, cleft palate surgery, and hypospadias surgery. This will surely take time, but will be a giant step toward better documentation. It is time to take this next step.

\section{Surajit Bhattacharya} Editor Indian Journal of Plastic Surgery E-mail: surajitbh@yahoo.co.in

\section{REFERENCES}

1. Available from: http://www.cebm.jr2.ox.ac.uk/docs/levels.html [Last accessed 2014 June 20].

2. Cochrane Injuries Group Albumin Reviewers. Human albumin administration in critically ill patients: Systematic review of randomised controlled trials. BMJ 1998;317:235-40.

3. Beauchamp T, Childress J. Principles of Biomedical Ethics. New York (NY): Oxford University Press; 2001.

How to cite this article: Bhattacharya S. Let's take the next step in documentation. Indian J Plast Surg 2014;47:157-8. 